SERIES DE CASOS

\title{
Embarazo abdominal a término: Reporte de un caso
}

\author{
Mario Díaz González*
}

Recibido: Julio 16/2001 - Revisado: Octubre 11/2001 - Aceptado: Febrero 22/2002

\section{RESUMEN}

El embarazo abdominal es poco frecuente y está asociado con alta mortalidad materna y fetal. Es raro que llegue al término y mucho más obtener un recién nacido en buenas condiciones.

Un caso de embarazo abdominal a término (40,5 semanas), el cual fue atendido exitosamente en la Clínica General del Norte en Barranquilla, Colombia por el Departamento de Ginecología y Obstetricia: Paciente de 33 años G2P1 con diagnóstico de ingreso de embarazo de 40,5 semanas, DPPNI y pre-eclampsia; a quien se le hizo un diagnóstico transoperatorio de embarazo abdominal a término, con producto de la gestación en buenas condiciones.

PALABRAS CLAVE: Embarazo abdominal, embarazo ectópico, pre-eclampsia, eclampsia, desprendimiento prematuro de placenta normoinserta.

\section{SUMMARY}

Abdominal pregnancy is uncommon and associated with higher fetal and maternal mortality. It is rare to grow to term and furthermore obtain a newborn in good conditions.

A case of abdominal pregnancy at term ( 40,5 weeks) which was successfully treated in the Clinica General del Norte, Barranquilla, Colombia, by the obstetrics and gynecologist department; was an obstetric patient of 33 years old, G2P1 with initial diagnosis of 40,5 weeks of gestation, abruptio placentae and pre-eclampsia; with postoperative diagnosis of abdominal pregnancy at term with a newborn in good conditions.

This article is a case report. It has a general interest for two reasons:

1) Its an uncommon event in obstetrics, the abdominal pregnancies with an alive fetus have very now frequency in the world.

2) A brief revision and how to confront this kind of cases is made.

KEY WORDS: Abdominal pregnancy, ectopic pregnancy, pre-eclampsia, eclampsia, abruptio placentae.

\section{INTRODUCCIÓN}

La incidencia del embarazo ectópico ha aumentado en las ultimas décadas, oscila * Gineco-Obstetra. Clínica General del Norte- Barranquilla,
Colombia. Profesor. Departamento de Gineco-Obstetricia.
Pregrado y postgrado - Universidad Metropolitana y entre los 4,5 y los 12,5 por 1000 embarazos ${ }^{1-4}$.

Hospital Universitario Metropolitano. Barranquilla, 
El embarazo ectópico se define como la implantación y desarrollo del óvulo fecundado fuera de la cavidad uterina; y de acuerdo a su localización puede ser: tubárico (97\%), abdominal (1-1.4\%), cervical $(0,1 \%)$, ovárico $(0,5 \%)$, Intramural $(0.6 \%)^{1-7}$. Es responsable del 9 al $14,2 \%$ de las muertes maternas en el primer trimestre del embarazo ${ }^{1,3}$. El embarazo abdominal es el que se desarrolla en las superficies peritoneales del abdomen y su frecuencia es de 1 en 3000 a 1 en 8000 partos $^{1-4}$.

Entre los factores de riesgo se mencionan: $\mathrm{La}$ enfermedad pélvica inflamatoria, cirugías tubáricas, dispositivos intrauterinos, promiscuidad, endometriosis, tratamientos para la fertilidad, anomalías uterinas, embarazo ectópico previo y el dietiletilbestrol ${ }^{1,3,8-9}$.

La mortalidad materna del embarazo ectópico abdominal es de 5 al 20\% y la viabilidad fetal es ocasional (10-25\%) con una frecuencia muy alta de anomalías fetales (35-75\%).

Para el diagnóstico son importante la clínica y el ultrasonido, aunque éste último en ocasiones puede omitir el diagnóstico e incluso ser desorientador $(50 \%$ de los casos) $^{8-9}$. Frecuentemente el diagnóstico no es sospechado y se hace durante el acto quirúrgico ${ }^{9}$.

\section{CASO CLÍNICO}

A continuación presento un caso de embarazo abdominal a término: Se trata de una paciente de 33 años, G2P1 con embarazo de 40,5 semanas, a quien se le realizó control prenatal en una institución de primer nivel con sus respectivos paraclínicos y ecografía a las 23 semanas que reportó embarazo intrauterino.

El día 6 de marzo del 2000 a las 3:00 a.m. la paciente en mención, ingresa a la sala de Urgencias de la Clínica General del Norte de Barranquilla remitida de un hospital de primer nivel con diagnóstico de pre-eclampsia severa, obito fetal y desprendimiento prematuro de placenta normoinserta (DPPNI).

\section{PLACENTA}
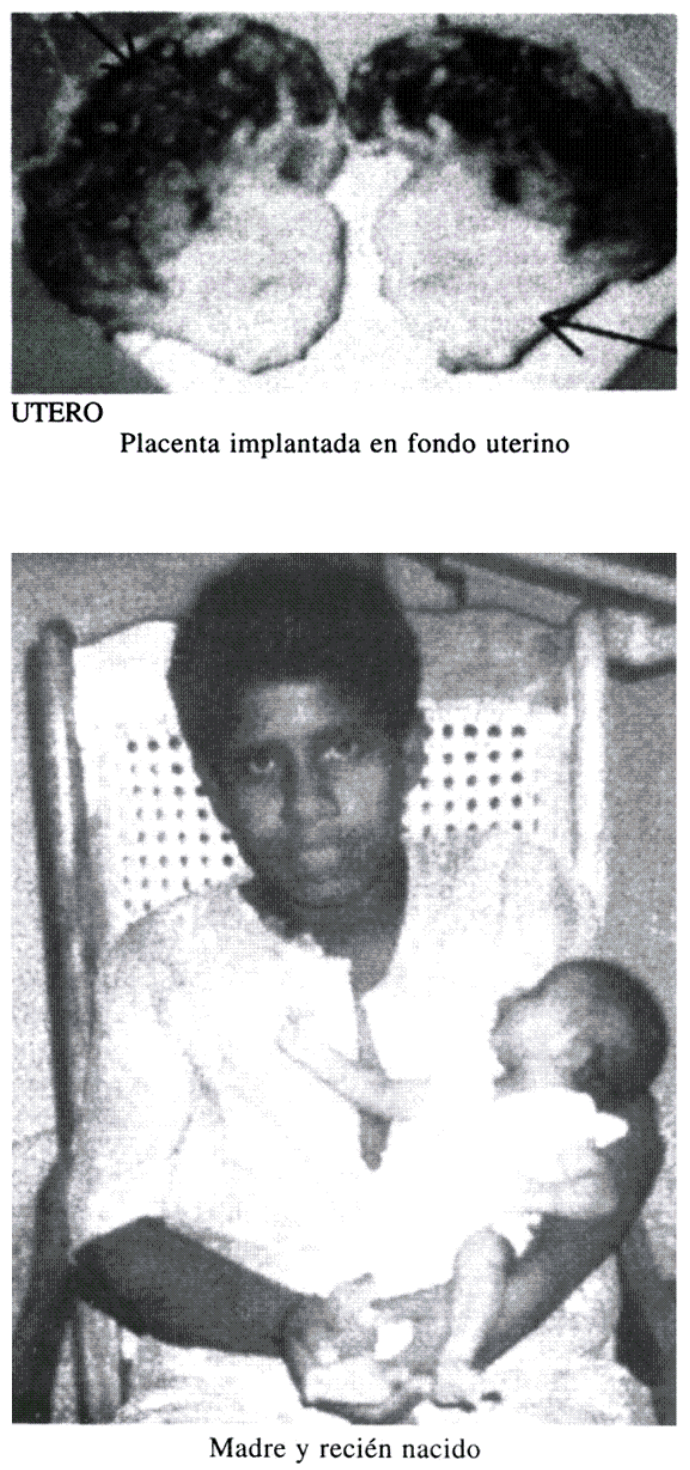

$\mathrm{Al}$ examen físico se encuentra pálida, hipotérmica, sudorosa, en estado de estupor, tensión arterial: 100/50 mmHg, Pulso: 140/minuto, frecuencia respiratoria: 24/minuto, abdomen con resistencia muscular y doloroso a la palpación, nose escucha fetocardia. No hay sangrado vaginal, cervix cerrado, no se palpa presentación.

La paciente es estabilizada hemodinámicamente y sometida a cirugía donde se encontró producto en cavidad abdominal, con membranas rotas, líquido amniótico meconial, placenta insertada en fondo uterino, desprendida en $15 \%$ de su área y hemoperitoneo de 500 cc. 
Se obtiene producto de sexo femenino con Apgar de 9/10 al minuto y 10/10 a los cinco minutos, peso 2900 gr, no presentó ninguna complicación. Se hizo histerectomía subtotal con placenta in situ por su estado general, acretismo y riesgo de mayor hemorragia.

La madre presentó eclampsia postparto, para lo cual se trasladó a unidad de cuidados intensivos (UCI) para tratamiento convencional (soporte ventilatorio, líquidos y electrolitos, difenilhidantoina, sulfato de magnesio, ampicilina y transfusión de 500 cc de sangre).

La paciente evolucionó satisfactoriamente.

La paciente fue dada de alta el 8 de marzo del 2000 con su hija en brazos.

El Diagnóstico definitivo fue:

1. Embarazo abdominal a término, con recién nacido vivo y peso adecuado para la edad gestacional.

2. Eclampsia

3. Desprendimiento parcial de placenta

\section{COMENTARIOS}

El embarazo abdominal avanzado es supremamente raro, ya que la mayoría de los fetos sucumben. Cuando se diagnostica después de las 24 semanas el tratamiento es expectante e intrahospitalario hasta esperar la viabilidad fetal; éste conlleva el riesgo de hemorragia súbita intra-abdominal que puede poner en peligro la vida de la madre. Si la gestación es menor de 24 semanas es adecuado el tratamiento quirúrgico con extracción del feto debido a que la supervivencia fetal es extremadamente baja $^{2,4-5,11}$.

Por lo anterior la incidencia del embarazo abdominal a término y exitoso es muy baja (1 de cada 25.000 nacimientos). En cuanto al manejo de la placenta la mayoría de los autores coinciden en extraerla si está implantada en un órgano no vital; de lo contrario se corta el cordón cerca a la placenta, cerrando posteriormente el abdomen.

La evolución placentaria puede monitorearse con ecografías y determinaciones seriadas de gonadotropina coriónica. También puede usarse metrotexate. En este caso se hizo histerectomía subtotal con placenta in situ, ya que ésta presentaba desprendimiento del $15 \%$ y acretismo, considerándose el tratamiento mas seguro $y$ sin riesgo posterior de hemorragia o abscesos intraabdominales.

El desarrollo sicomotor y pondoestatural del producto de este embarazo hasta el momento ha sido totalmente normal.

\section{BIBLIOGRAFÍA}

1. Pisarska MD. y Carson, SA. Embarazo ectópico. Clínicas Obstétricas y Ginecológicas. Editorial Interamericana México. 1999;1: 3-7.

2. Uzandizaga, J.A., De la Fuente, P. Tratado de Obstetricia y Ginecología. 1 ed. Madrid (España): Editorial Interamericana S.A.; 1998. p. 266-76.

3. Te Linde, Thompson R. Ginecología Quirúrgica. 7 ed. Buenos Aires (Argentina) Editorial Médica Panamericana. 1998. p. 521-43.

4. González Merlo, J. Obstetricia. 4 ed. Barcelona (España): Editorial Masson-Salvat. p. 330-37.

5. Jaramillo EL. Embarazo Abdominal: análisis de una "epidemia". Rev Colomb Obstet Ginecol 1998;49:97-100.

6. Pérez Sánchez, A. Ginecología. 2ed. Santiago (Chile): Editorial Publicaciones Técnicas Mediterráneo; 1995. p. 230-44.

7. Martínez M. Embarazo ectópico. Informe de 82 casos. Hospital U. Metropolitano. Rev Colomb Obstet Ginecol 1999;50:244-6.
8. García Mazorra M. Morera O. Embarazo abdominal. "Enrique Cabrera". La Habana. Disponible en: http://www.perinat.org.ar/embarazo_abdominal.htm 9. Messa Acosta, A. Embarazo Abdominal Caso Clínico y Revisión. Disponible en: http://www.geocities.com/HotSprings/Resort/4015/ab dominal.html

10. Acosta Osio G. Tratado de Ginecología y Obstetricia. 2 ed. Barranquilla (Colombia): Editorial Espriellabe y Asociados; 1992. p. 66-69.

11. Cunningham MG, MacDonald G. Williams Obstetricia. 4 ed. Barcelona (España): Editorial Masson S.A.; 1996.

12. Ñanez $H$, Ruiz A. Texto de Obstetricia $y$ Ginecología. 1 ed. Bogotá (Colombia): Universidad Nacional de Colombia. 1999. p. 244-46. 